\author{
Military Technical College \\ Kobry El-Kobbah, \\ Cairo, Egypt.
}

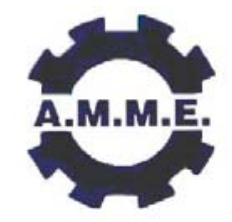

\author{
$13^{\text {th }}$ International Conference \\ on Applied Mechanics and \\ Mechanical Engineering.
}

\title{
OPTIMIZATION SCHEME for a SMALL CROSS-FLOW HYDRO TURBINE RUNNER
}

\author{
AKCAN $^{\star}$ C., AKSIT ${ }^{\dagger}$ M. F., KIZILTAS ${ }^{\ddagger}$ G. and KANDEMIR ${ }^{\S}$ I.
}

\begin{abstract}
Obtaining a closed form analytical formulation for complex mechanical structures such as turbine rotors with multiple blade assemblies can be very problematic. Building a prototype without optimizing the structural stress levels may result in unnecessary hardware costs. In this work, mathematical models for highest stress or lowest factor of safety on critical components of a small modular cross-flow hydro turbine rotor have been explored for various design options through a response surface analysis. The response surface model is obtained based on finite element analysis results following design of simulated experiments. Rotor design parameters have been investigated to optimize factor of safety on critical components without violating the specified weight limits. Box-Henken data tables have been used to obtain the response surface model. The attained response model yields the maximum stress and lowest factor of safety for critical rotor components for various combinations of design parameters. Verification runs indicate that response model successfully predicts factor of safety levels close to finite element calculations.
\end{abstract}

\section{KEY WORDS}

Hydraulic machinery, turbine, multidisciplinary design, optimization, response surface methodology, sequential quadratic programming, genetic algorithm.

\footnotetext{
* Graduate student, Faculty of Engineering and Natural Sciences, Sabanci University, Istanbul, Turkey

Associate professor, Faculty of Engineering and Natural Sciences, Sabanci University, Istanbul, Turkey

* Assistant professor, Faculty of Engineering and Natural Sciences, Sabanci University, Istanbul, Turkey

$\S$ Assistant professor, Design and Manufacturing Engineering, Gebze Institute of Technology, Kocaeli, Turkey
} 


\section{INTRODUCTION}

Around the world, only a small portion of the available energy in small rivers is utilized for electric power generation. Although the power regimes in such small rivers fluctuate a great deal, stored energy accounts for a considerable amount for the human use. The available energy can be in the form of a head (drop in elevation), or flow rate. The combination of these two factors will define the total hydraulic energy potential. Depending on the form of the hydro-energy available, different power turbine types are used to convert this hydraulic potential to electricity most efficiently. Commonly used hydro-turbines can be named as Pelton, Francis, Kaplan and cross-flow turbines. Cross-flow turbines are also known as Banki or Ossberger turbines. Figure 1 illustrates [1] suitable application ranges for various turbine types based on available head and flow rates. Cross-flow turbines are only suited for small power applications typically less than $1000 \mathrm{~kW}$.

A cross-flow turbine consists of a cylindrical water wheel with a horizontal shaft, composed of numerous radially and tangentially arranged blades. The ends of the blades are welded to disks, spaced equally in the axial direction. A cross sectional view of cross-flow turbine is illustrated in Fig. 2 [2]. Thus, the water energy is transferred to the rotor blades twice in a single cycle [3].

For years, cross-flow turbines did not attract any interest due to the small power range and relatively lower efficiency rates. Therefore, there is very limited published work on these turbines. Research is needed to increase efficiency of these turbines while decreasing their cost. In this work, cross-flow turbines are analyzed. Two well known optimization algorithms, Sequential Quadratic Programming (SQP) and Genetic Algorithms (GA), are applied. Stress distributions on critical rotor components are numerically obtained using Finite Element Analysis. Then a mathematical model of the stress distributions has been obtained through response surface analysis. Optimum design parameters for the highest factor or safety or the lowest stress levels on the critical rotor components are reported.

Although it is difficult to find available literature for cross flow turbines and their rotor stress levels, there are works available for various other turbine types. A former work by Guoyi et. al. [4, 5] dealt with the inverse design optimization of hydraulic turbine runner blade geometry in Kaplan turbines. Dornberger et. al. [6] developed an optimization environment for multidisciplinary turbomachinery blade design. He proposed a framework for the parallel execution of the solver computations. Egartner and Schulz [7] developed a partially reduced SQP algorithm that makes way for an efficient parallel implementation of blade design optimization. Similarly, Schulz presented a work on cross sectional shape optimization of a turbine blade [8].

In this work, rather than focusing only on the blade geometry, an optimization scheme has been outlined to determine critical components on the runner of a cross-flow turbine and to optimize the factor of safety on these components. The paper is structured as follows. First a brief explanation of modular cross-flow hydro turbine runner structure is given. Then, an objective function is derived with all constraints for the optimization problem. The results for the applied optimization algorithms are presented. The optimum design vector for the highest factor of safety on critical runner components is obtained. Finally, a discussion on the effectiveness of the applied optimization scheme has been presented. 


\section{CROSS-FLOW TURBINE RUNNER STRUCTURE}

As illustrated in Figures 2 through 4, a cross-flow turbine runner is made of multitude of blades. Tangential blades are mounted on radial support disks that are evenly spaced in runner axial direction. As each blade has to be secured on support disks, runner assembly requires a lot of welding work. A modular runner structure is needed to reduce custom design and fabrication time as well as to minimize required welding work. The focus of this paper is to develop and present a working optimization scheme for the runner stresses rather than the structural design details. A parallel effort is being undertaken to develop a modular cross-flow turbine design which introduces structural flexibility in the construction and installation periods. This work will be presented in another paper.

\section{OBJECTIVE FUNCTION}

Selection of design variables and the objective function for the turbine runner optimization should be implemented with care. At the preliminary stage, many design variables can be considered for the turbine runner construction. However, some typical values for most of these variables are commonly set to achieve the highest efficiency. In addition, some of these variables are interdependent. For example, optimum number of blades on a runner assembly depends on the runner size. Similarly, the radial blade spacing and blade thickness values are all determined in accordance with the rotor diameter. Likewise, blade entrance angle and length of the blade arc are all preset to maintain proper tangential contact between the water jet and blade entrance [3]. All of the preconditions are specified based on rough analysis to achieve the highest turbine efficiency. Hence, we decided to perform an optimization on the design variables of the cross flow turbine to minimize the stress distributions without affecting the overall turbine efficiency. However, a turbine runner has many elements manufactured from different materials. Therefore, a component with lower stress distribution does not always mean that the component is safe statically. Factor of safety analysis on a specific component is a better means to illustrate the endurance of the concerned component under the prescribed conditions. In brief, not taking into account any constraints, the objective function of our problem is to optimize the factor of safety on the critical components of the turbine runner.

To specify design vector for the above-mentioned objective function, first, it is required to detect the turbine parameters consistent with the maximum efficiency conditions (Table 1). The parameter values in Table 1 are derived with respect to the flow conditions for a typical low power (less than $30 \mathrm{~kW}$ ) cross-flow turbine. Therefore, without affecting the overall efficiency, we are left with three design variables which are disc thickness, disc spacing and shaft diameter (Fig. 4).

Available hydraulic energy in the form of head and flow rate is transferred to the turbine runner while water jet runs through blades. Therefore, instead of head and flow rate, the water force acting on the blades is taken as a parameter in the analysis. The power of the water jet acting on the turbine runner can be presented as [9],

$\dot{W}_{\text {shaft }}=T_{\text {shaft }} \omega=\dot{m} U(U-V)(1-\cos \beta)$ 
where

$\dot{W}_{\text {shaft }}:=$ shaft power

$T_{\text {shaft }}:=$ shaft torque

$\omega:=$ angular velocity of the runner

$\dot{m}:=$ mass flow rate

$U:=$ linear velocity of the runner at the blades

$V:=$ water jet velocity

$\beta:=$ entrance angle of the water jet

The values of these parameters are illustrated in the master table (Table 1). In addition, shaft torque can be formulated as,

$T_{\text {shaft }}=F . r$

where

$F:=$ impact force of the water jet

$r:=$ radius of the runner

In order to take the worst case conditions in consideration maximum torque that might be applied on the turbine runner is selected as the driving torque through our analysis. Maximum torque on the turbine occurs when the turbine is stalled. No power is transferred to the runner shaft because of the fact that its angular velocity is zero. Similarly, maximum torque gives way to the existence of maximum force on the turbine blades owing to the proportional relationship with runner radius. By taking the velocity of runner $(U)$ zero and enforcing the velocity of the water jet and water entrance angle according to maximum efficiency conditions (Table 1), the maximum torque applied on the turbine blades is calculated. Likewise, by introducing the runner radius, maximum force acting on the turbine blades is obtained for the selected operating conditions around $7500 \mathrm{~N}$. For a conservative analysis this force is assumed to act only on a single blade along the runner. All of the analyses and optimization runs are based on this approach.

In addition to the design parameters mentioned above, disk type, blade and shaft materials are also other important design factors. Disk and blades are manufactured from AISI 1023 Carbon Steel and the shaft is manufactured from annealed 4340 Alloy Steel. These two material characteristics are also added to the problem as additional parameters.

As stated earlier, the optimization for factor of safety is aimed to be performed on the critical component of the turbine runner. A number of finite element analysis (FEA) simulations are conducted as part of a screening process to identify most critically loaded runner components under various operating conditions. Screening results pointed out that the most critical component on the runner is the disc attached to the short side of the shaft.

In order to define the objective function of the optimization problem completely, some constraints should be enforced into the problem. A turbine design with thicker disc sand 
blades or with larger shaft radius would result in the highest factor of safety. However, the output power to weight ratio of the turbine is also an important parameter for overall efficiency and cost. In other words, it is an important requirement for the turbine designer to minimize the runner weight as much as possible. Therefore, adding a weight constraint is necessary for proper handling of this optimization problem. Taking typical turbine design conditions into consideration, a turbine weight around $50 \mathrm{~kg}$ is a reasonable weight constraint. Also, three design variables, i.e. disc thickness, disc spacing and shaft diameter, have upper and lower bounds to keep the overall turbine design in feasible region (Table 2). Overall, the final objective of the optimization problem is defined as follows. Optimize the factor of safety on the disc at the short side of the runner according to static analysis results by optimizing the disc thickness, disc spacing and shaft diameter in the feasible region without exceeding the weight limits.

Similarly, the formal problem statement can be summarized theoretically as,

$$
\begin{gathered}
\min (-f o s(\mathbf{x}, \mathbf{p})) \\
\text { subject to: } \\
\text { weight }(\mathbf{x}, \mathbf{p}) \leq 50 \mathrm{~kg} \\
\text { along with the boundary conditions }(\mathrm{mm}) \text { : } \\
2 \leq x 1 \leq 8 \\
58 \leq x 2 \leq 174 \\
40 \leq x 3 \leq 60 \\
\text { where } \\
\mathbf{x}=\left[\begin{array}{l}
x 1 \\
x 2 \\
x 3
\end{array}\right]:=\left[\begin{array}{l}
\text { disc thickness } \\
\text { disc spacing } \\
\text { shaft diameter }
\end{array}\right]
\end{gathered}
$$

As it is noticed in the formal problem statement, the factor of safety optimization problem is modified into a minimization problem by taking the negative of the objective function. Moreover, the upper bound for the weight constraint is set as $50 \mathrm{~kg}$. This boundary is not a strict boundary because of the fact that the weight requirement of the real turbine is not determined in specific values.

\section{APPLIED OPTIMIZATION ALGORITHMS and ANALYSIS RESULTS}

The objective function is a nonlinear function due to the fact that design variables (disk thickness, disc spacing and shaft diameter) have coupled effects on factor of safety (FOS) for each component in the runner. The optimization problem is further constrained by the weight restriction. Therefore, optimization algorithms which are capable of handling constrained nonlinear problems are required for solution. Sequential quadratic programming (SQP) for convex problems [10] and genetic algorithm [11], which is a special heuristic technique, are well known optimization algorithms for dealing with such nonlinear problems. 
During optimization process each step requires a new finite element analysis (FEA) for the turbine runner. For example, in sequential quadratic programming technique, the search direction towards the optimum is updated in each step, and design variables are assigned to new values. To get the output of objective function in each step, running a new design scenario with the updated design variables is necessary. This is analogous to avoiding the task of manufacturing and assembling a new runner in each step and making experiments on the runner to get the stress output which is extremely long and costly process. Similarly, running finite element analysis for each set of design variables is also time consuming and requires powerful computational resources. Rather than running lengthy computer simulations, it is commonly preferred to utilize simplified transfer functions to conduct optimization process. Therefore, based on finite element analyses results response surface models [12] are generated and used for runner stress optimization process.

Response surface models are multivariate polynomial models and typically arise in tdesign of experiments (DOE) [13], where they are used to determine a set of design variables that optimize a response. For multivariate optimization problems having a large set of design variables, a factorial experiment can be implemented to reduce the number of design variables. Most of the time, it becomes sufficient to determine which variables have a significant impact on the objective function. Then, a central composite design [14] or a Box-Behnken design [15] can be implemented to obtain a convex polynomial, which is an approximation at best. However, the ease of estimation and application of the model, even when little is known about the process, makes it a preferable choice among optimization techniques.

\section{Box-Behnken Design}

Box-Behnken designs are response surface designs that can fit a full quadratic model. Such designs use three level for each factor: one at the upper bound, one at the lower bound and one at the middle. Moreover, Box-Behnken designs are successful for fitting quadratic models when the number of design variables is relatively low which is suited to our problem which has only three design variables; disc thickness, disc spacing and shaft diameter.

Figure 5 shows a Box-Behnken design for three factors [16], with the circled point appearing at the origin and possibly repeated for several runs. The replicated center point runs allow us to estimate the prediction variance more uniformly over the entire design space.

For our factor of safety (FOS) optimization problem, we have 3 design variables which are suitable for implementing Box-Behnken design approach, as the number of design variables is relatively low. Box-Behnken data set (Table 3) for our problem has been formed using a commercially available computer code, JMP, which can perform simple and complex statistical analyses. Each runner component has been designed using commercially available computer code, Solidworks. Once 3-D solid models are generated, these files are imported into another commercially available analysis code, Cosmosworks, to perform detailed finite element analyses. The set values "0", "-" and "+" in Table 3 stand for middle point, lower bound and upper bound, respectively for each design variable. Then stress and factor of safety values for the disc attached to the short side of the shaft are obtained, and corresponding weight values for the runner are presented in Table 3. All of the calculations are based on the static finite element 
analysis results obtained from the Cosmosworks. The predicted values for the FOS and weight parameters are obtained from the optimization routine from JMP code that fits a convex second-degree polynomial function. The results are presented as the additional two columns in Table 3.

Because stress and factor of safety results reflect solutions of analyses that are run on a finite element computer code rather than reflecting actual experimental measurements, repeating the analyses for the same variable set does not yield different results. Therefore, the design combination representing the center point is analyzed only once. Based on predicted values, the nonlinear quadratic objective and constraint functions have been obtained as follows.

Weight $=$

$4.151 a-.1676 b+0.02295 c-0.01625 a b-0.00258 a c+0.000105 b c-0.00065 a^{2}+0.0039 c^{2}+0.0007 b^{2}+38.67(3)$

FOS $=$

$0.4614 \mathrm{a}+0.02948 b+0.1398 c+0.00029 a b+0.01442 a c-0.0002 b c-0.09289 a^{2}-0.000465 c^{2}-0.000093 b^{2}-6.159(4)$

where a, b and c stand for 'disc thickness', 'disc spacing' and 'shaft diameter', respectively.

\section{Sequential Quadratic Programming}

Sequential Quadratic Programming (SQP) is considered to be the best gradient based algorithm and widely used in many engineering applications [10]. SQP methods attempt to solve a nonlinear program by constructing and solving a local model of the optimization problem at each step. If the SQP method is not trapped by a local minimum, it yields the solution of the original problem. Since our optimization problem is a constrained nonlinear problem, as the first choice, we applied SQP method to get the optimum design vector for the highest factor of safety on the critical disc component. A sequential programming code has been generated implementing both constraint and objective functions. The code is a total reflection of the formal problem statement illustrated earlier in the objective function section.

The starting design vector for the optimization problem is stated as follows.

$\mathbf{x}_{i}=\left[\begin{array}{l}x l_{i} \\ x 2_{i} \\ x 3_{i}\end{array}\right]:=\left[\begin{array}{l}\text { initial disc thickness } \\ \text { initial disc spacing } \\ \text { initial shaft diameter }\end{array}\right]=\left[\begin{array}{l}7 \mathrm{~mm} \\ 75 \mathrm{~mm} \\ 45 \mathrm{~mm}\end{array}\right]$

Although the initial values for design vector are in feasible region, diagnostics of SQP algorithm reports that the starting point is out of feasible region. This is because of the fact that the turbine runner weighs $59 \mathrm{~kg}$ for this initial design vector, and it violates the inequality constraint which is set as $50 \mathrm{~kg}$. For various initial starting points, which are in feasible design space, the same factor of safety result is obtained. Optimum design parameters and the corresponding factor of safety on the most critically loaded component are obtained as follows. 
$p=$

$\mathrm{FOS}=$

\section{$-4.6245$}

Figure 6 shows the maximum factor of safety value converged at each iteration (negative values are shown as the problem is formulated as a minimization problem). Running various other optimization sets validate that the starting point does not change the optimum obtained for the problem. For example, if the starting point of the algorithm is chosen as $x l_{i}=4.5 \mathrm{~mm}, x 2_{i}=140 \mathrm{~mm}$ and $x 3_{i}=50 \mathrm{~mm}$, the factor of safety still converges to 4.6245 . The optimized design variables becomes,

$\mathbf{x}_{\text {opt }}=\left[\begin{array}{l}x 1_{\text {opt }} \\ x 2_{\text {opt }} \\ x 3_{\text {opt }}\end{array}\right]:=\left[\begin{array}{l}\text { optimum disc thickness } \\ \text { optimum disc spacing } \\ \text { optimum shaft diameter }\end{array}\right]=\left[\begin{array}{l}4.5269 \mathrm{~mm} \\ 147.6324 \mathrm{~mm} \\ 54.1361 \mathrm{~mm}\end{array}\right]$

Converging to the same optimum design variables for various starting points is a wellknown common characteristic of a function that has no local minimums. Moreover, the weight inequality constraint is active in the problem. In other words, the increase of the factor of safety to a higher value is limited by the weight constraint. Even if the specifications of sequential quadratic programming solver are changed, the optimum design vector converges to the same value.

On the other hand, expanding the constraint boundary affects the optimum design variables because of the fact that weight is an active constraint in this problem in any case. For instance, if the weight constraint is increased to $60 \mathrm{~kg}$, the optimum design variables are calculated as follows.

$\mathbf{x}_{\text {opt }}=\left[\begin{array}{l}x 1_{\text {opt }} \\ x 2_{\text {opt }} \\ x 3_{\text {opt }}\end{array}\right]:=\left[\begin{array}{l}\text { optimum disc thickness } \\ \text { optimum disc spacing } \\ \text { optimum shaft diameter }\end{array}\right]=\left[\begin{array}{l}7.1068 \mathrm{~mm} \\ 114.1499 \mathrm{~mm} \\ 60 \mathrm{~mm}\end{array}\right]$

Figure 7 presents the evaluation of the algorithm for this case. The new optimum design vector and factor of safety values indicate that weight constraint is the limiting factor in this problem. This is expected based on the physics of the problem. The increase in the weight constraint results in an increase in the minimum factor of safety to 6.2575. Furthermore, this time, shaft diameter restriction is at the upper bound and is active.

\section{Genetic Algorithm}

The second algorithm implemented to find the optimum design variables is a genetic algorithm (GA) [11], arises in the class of heuristic methods. Main motivation for heuristic methods is to deal with local minima and not get trapped in them while searching for the global optimum. Moreover, heuristics are used to solve complex multivariate optimization problems that are extremely challenging. Our objective 
function is a convex function which ensures that it has no local minimums in the problem. Thus, we have implemented genetic algorithm to observe its performance on convex shape polynomials and compare the performance with the results from sequential quadratic method. Since the original problem is rather simplified by a convex quadratic polynomial obtained from response surface methodology, no matter how parameters of genetic algorithm are modified (such as the population size, mutation rate, selection or crossover rates), the optimum result is found around the values obtained from the gradient based technique. Moreover, it appears that the heuristic method operates very quickly and converges to the correct result.

The genetic algorithm parameter set defined in Matlab optimization toolbox is presented below. In each example, objective function is constrained by an upper weight limit adjusted to $60 \mathrm{~kg}$. Since our problem is defined by a quadratic function, constraints can not change the function's characteristic, that is to say, there is one local minima in any case. Therefore, in this part, objective function is optimized only for a weight limit adjusted to $60 \mathrm{~kg}$. Convergence history of the genetic algorithm ensures that the objective function would converge to the same factor of safety for any weight limit as in the case of SQP. The observation in this part is based on the comparison between the convergence speeds of both algorithms. It seems that genetic algorithm converges faster than the SQP algorithm, which is advantageous for large scale problems.

Optimization example 1

$\mathbf{x}_{\text {opt }}=\left[\begin{array}{l}x l_{\text {opt }} \\ x 2_{\text {opt }} \\ x 3_{\text {opt }}\end{array}\right]:=\left[\begin{array}{l}\text { optimum disc thickness } \\ \text { optimum disc spacing } \\ \text { optimum shaft diameter }\end{array}\right]=\left[\begin{array}{l}6.98427 \mathrm{~mm} \\ 111.9283 \mathrm{~mm} \\ 59.99 \mathrm{~mm}\end{array}\right]$

Fitness function value, FOS $=6.25555$ (Fig. 8)

Weight $=60 \mathrm{~kg}$

Optimization example 2

The convergence criteria of genetic algorithm is reconfigured with different settings.

$\mathbf{x}_{\text {opt }}=\left[\begin{array}{l}x l_{\text {opt }} \\ x 2_{\text {opt }} \\ x 3_{\text {opt }}\end{array}\right]:=\left[\begin{array}{l}\text { optimum disc thickness } \\ \text { optimum disc spacing } \\ \text { optimum shaft diameter }\end{array}\right]=\left[\begin{array}{l}7.1018 \mathrm{~mm} \\ 114.0549 \mathrm{~mm} \\ 59.99 \mathrm{~mm}\end{array}\right]$

Fitness function value, FOS $=6.257227$ (Fig. 9)

Weight $=60 \mathrm{~kg}$

As it is noticed from the results, no significant change occurs when the GA parameters are modified. The results are close to the SQP results. GA algorithm solution also confirms the absence of a local minimum in the feasible region of the design space.

\section{Numerical Validation}

As stated earlier, second order convex polynomial obtained from response surface methodology is an approximation to the original problem. Thus, validation of optimum design vector through finite element analysis is a major part in this work. In this section, 
factor of safety results obtained from finite element analysis and from second order convex function of response surface model are compared.

First of all, a new runner design is created in Solidworks with the optimum design parameters for the problem constrained by $50 \mathrm{~kg}$. After that, the model is loaded with the same force, which is $7500 \mathrm{~N}$. Then, the model is meshed with the same elements and analyzed. However, the disc spacing value in the optimum design vector can not be directly implemented in the new runner design due to the fact that the length and the diameter of a runner are determined according to the hydraulic power potential of the application. Hence, the length of the turbine shaft needed to be modified in order to match the design geometry. Because of the symmetric consistency, it is required to distribute the discs on the shaft equally. In order to place equally spaced discs on the shaft, we have to modify disc spacing data obtained from optimization algorithm, to a near value that is dividing the shaft length equally. Thus, the disc spacing data is changed from $147.6324 \mathrm{~mm}$ to $178 \mathrm{~mm}$ in the optimum design set. The model is recreated and analyzed again accordingly. Safety factor on the critical disc then becomes 4.436 (Fig. 10). On the other hand, quadratic function giving the factor of the safety of the critical disc yields 4.3507 when the optimum design parameters with the modified disc spacing value entered. Taking into account that response surface model gives only an approximation to the original problem, the results are in good agreement for this case.

The second comparison is between the factor of safety results of response surface model and the results of the finite element analysis when the weight limit is increased to $60 \mathrm{~kg}$. Again, a new runner design with optimum design parameters is generated. In this case, actual value of optimum disc spacing $114.1499 \mathrm{~mm}$ is changed to $116 \mathrm{~mm}$ which is dividing the shaft length into 3 equal parts. Minimum factor of safety on the critical disc obtained from finite element analysis is 6.085 (Fig. 11). Factor of safety result obtained from response surface function is 6.2576. Although the results are not as close as in the previous case, response surface model mimics the stress distribution on the critical disc successfully. As a result, it is clear that the static stress distribution on the critical disc can be monitored effectively by response surface methodology.

\section{CONCLUSION}

Through response surface methodology, design optimization of the cross-flow hydro turbine has been investigated. The results of SQP and GA algorithms converge to the same global optimum. The convergence speeds of both algorithms are very good that the results are obtained very fast without convergence problems.

It has been demonstrated that response surface models can effectively represent transfer functions for complex structures. Moreover, common optimization techniques like SQP and GA apply well to the quadratic functions obtained from the response surface model. Although GA is converging faster than SQP, it is not a considerable amount of time for small scale problems as our case.

When the optimum design variable vector, which is constrained by a weight limit of 50 $\mathrm{kg}$., is observed, an optimal length of $147.6323 \mathrm{~mm}$. for disc spacing is obtained by the SQP algorithm. However, when the width of the turbine is considered as a constant parameter, it seems that equal distribution of discs for every measurement of length is 
impossible. Therefore, as a further study, integer programming techniques can be applied to cope with this problem. On the other hand, the optimization problem can take a multi-objective form by enforcing a lower limit on the factor of safety of each component on the runner, as well. Each of these factor of safety limits can be stated as separate objective functions that transforms the problem into a multi-objective one. A weighted approach technique may also be applied to form a multi-objective function incorporating the characteristics of all individual objectives.

\section{REFERENCES}

[1] A Guide to UK Mini-Hydro Developments: The British Hydropower Association, 2005.

[2] C. Penche, Layman's Guidebook on How to Develop a Small Hydro Site, Second ed: Commission of the European Communities, 1998.

[3] C. A. Mockmore and F. Merryfield, "The Banki Water Turbine," Engineering Experiment Station(of Oregon State College), vol. Bulletin Series No. 25, 1949.

[4] G. Peng, S. Cao, M. Ishizuka, and S. Hayama, "Design optimization of axial Flow hydraulic turbine runner: Part I-an improved Q3D inverse method," International Journal for Numerical Methods in Fluids, vol. 39, pp. 517 - 531, 2002.

[5] G. Peng, S. Cao, M. Ishizuka, and S. Hayama, "Design optimization of axial Flow hydraulic turbine runner: Part II-multi-objective constrained optimization method," International Journal for Numerical Methods in Fluids, vol. 39, pp. 533 548, 2002.

[6] R. Dornberger, P. Stoll, D. Büche, and A. Neu, "Multidisciplinary Turbomachinery Blade Design Optimization," AIAA, vol. 0838, 2000.

[7] W. Egartner and V. H. Schulz, "Partially reduced SQP methods for optimal turbine and compressor blade design," presented at 2nd European Conference on Numerical Mathematics and Advanced Applications, ENUMATH 97, Singapore, 1998.

[8] V. H. Schulz, "Numerical optimization of the cross-sectional shape of turbine blades," ZAMM, vol. 76, pp. 207 - 210, 1996.

[9] B. R. Munson, D. F. Young, and T. H. Okiishi, Fundamentals of Fluid Mechanics, 5 ed. New York; Chichester: Wiley, 2005.

[10] G. N. Vanderplaats, "Numerical Optimization Techniques for Engineering Design," Vanderplaats R\&D, 1999.

[11] D. E. Goldberg, Genetic Algorithms in Search, Optimization and Machine Learning: Addison-Wesley Publishing Company Inc., 1989.

[12] G. E. P. Box and K. B. Wilson, "On the Experimental Attainment of Optimum Conditions (with discussion)," Journal of the Royal Statistical Society, vol. 13, pp. $1-45,1951$.

[13] G. E. Box, W. G. Hunter, and J. S. Hunter, Statistics for Experimenters: Design, Innovation, and Discovery, 2 ed: Wiley, 2005.

[14] R. H. Myers, Response Surface Methodology. Boston: Allyn and Bacon, Inc., 1971.

[15] G. Box and D. Behnken, "Some new three level designs for the study of quantitative variables," Technometrics, vol. 2, pp. 455 - 475, 1960.

[16] Optimization Toolbox Help: Matlab, 2006. 


\section{TABLES}

Table 1. Master table displaying the parameters and variables

\begin{tabular}{|c|c|c|c|c|c|c|}
\hline & \multicolumn{6}{|c|}{ MASTER TABLE } \\
\hline & Definition & Symbol & L.B. & U.B. & constant & Unit \\
\hline \multirow{3}{*}{$\begin{array}{l}\frac{\mathscr{d}}{0} \\
\frac{\sqrt[0]{0}}{\frac{\pi}{\pi}} \\
>\end{array}$} & Disc Thickness & D.T & & & & $\mathrm{mm}$ \\
\hline & Disc Spacing & D.S. & & & & $\mathrm{mm}$ \\
\hline & Shaft Diameter & S. Dia & & & & $\mathrm{mm}$ \\
\hline \multirow{11}{*}{$\begin{array}{l}\frac{\omega}{\Phi} \\
\frac{\omega}{0} \\
\frac{c}{\pi} \\
\frac{\pi}{\sigma} \\
0\end{array}$} & Head & $\mathrm{Ho}$ & & & 8 & $\mathrm{~m}$ \\
\hline & Flow rate & Q & & & 300 & It $/ \mathrm{s}$ \\
\hline & Rotor Diameter & $\mathrm{D}$ & & & 300 & $\mathrm{~mm}$ \\
\hline & Rotor Width & B & & & 388 & $\mathrm{~mm}$ \\
\hline & $\begin{array}{l}\text { Water Entrance } \\
\text { Angle }\end{array}$ & $\beta$ & & & 16 & deg \\
\hline & Velocity of Rotor & $U$ & & & 0 & $\mathrm{~m} / \mathrm{s}$ \\
\hline & Velocity of Water Jet & $\mathrm{V}$ & & & 12,3 & $\mathrm{~m} / \mathrm{s}$ \\
\hline & \# of Blades & Z & & & 30 & \\
\hline & Force & $\mathrm{F}$ & & & 7500 & $\mathrm{~N}$ \\
\hline & Steel 4340 Annealed & & & & & \\
\hline & Steel 1023 Carbon & & & & & \\
\hline Objective F. & $\min (-F O S)$ & & & & & \\
\hline Constraint & Weight & W & & 50 & & $\mathrm{~kg}$ \\
\hline
\end{tabular}

Table 2. Bounds of the design variables

\begin{tabular}{|l|r|r|}
\hline Design Variable & Lower Bound & Upper Bound \\
\hline disc thickness & 2 & 8 \\
\hline disc spacing & 58 & 174 \\
\hline shaft diameter & 40 & 60 \\
\hline
\end{tabular}

Table 3. Box-Behnken Data Table

\begin{tabular}{|lrrrrrrr|}
\hline Pattern & D.T. & D.S. & S.Dia & FOS & weight & Pred Formula weight & Pred Formula FOS \\
$0--$ & 5 & 58 & 40 & 2,58 & 54,24 & 54,2235 & 2,5565 \\
-0 & 2 & 58 & 50 & 2,562 & 47,97 & 48,673625 & 2,49025 \\
$-0-$ & 2 & 116 & 40 & 1,57 & 41,314 & 40,626875 & 1,66525 \\
-+0 & 2 & 174 & 50 & 2,39 & 44,21 & 44,918375 & 2,26875 \\
$+0-$ & 8 & 116 & 40 & 2,62 & 52,84 & 53,564875 & 2,52225 \\
000 & 5 & 116 & 50 & 4,39 & 50,838 & 50,838 & 4,39 \\
$-0+$ & 2 & 116 & 60 & 3,53 & 49,76 & 49,035125 & 3,62775 \\
++0 & 8 & 174 & 50 & 4,02 & 52,75 & 52,046375 & 4,09175 \\
$0-+$ & 5 & 58 & 60 & 5,65 & 62,333 & 62,35425 & 5,624 \\
+-0 & 5 & 58 & 50 & 3,99 & 67,82 & 67,111625 & 4,11125 \\
$0+-$ & 5 & 174 & 40 & 2,65 & 44,712 & 44,69075 & 2,676 \\
$+0+$ & 8 & 116 & 60 & 6,31 & 60,976 & 61,663125 & 6,21475 \\
$0++$ & 5 & 174 & 60 & 5,24 & 53,05 & 53,0665 & 5,2635 \\
\hline
\end{tabular}




\section{FIGURES}

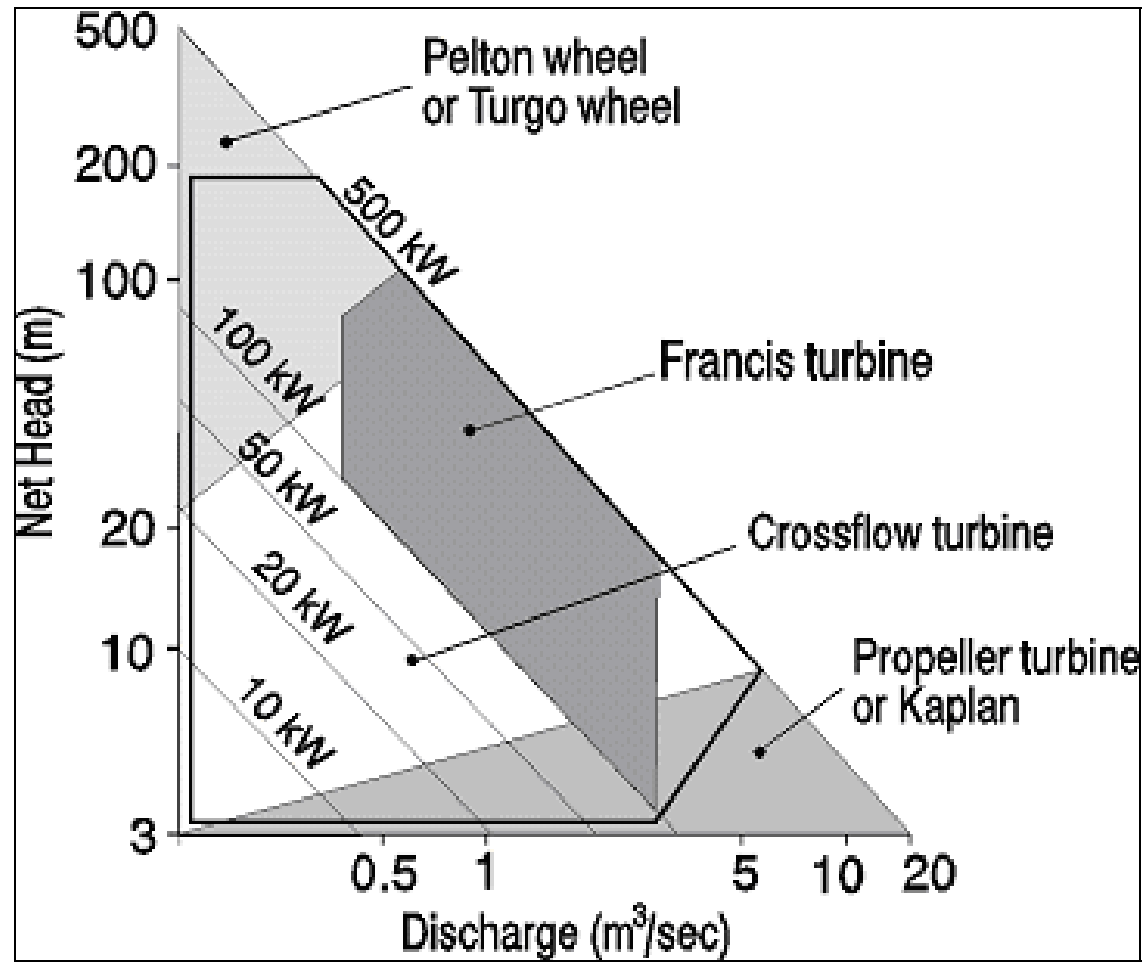

Fig.1. Head-Flow Ranges of Small Hydro Turbines. [1]

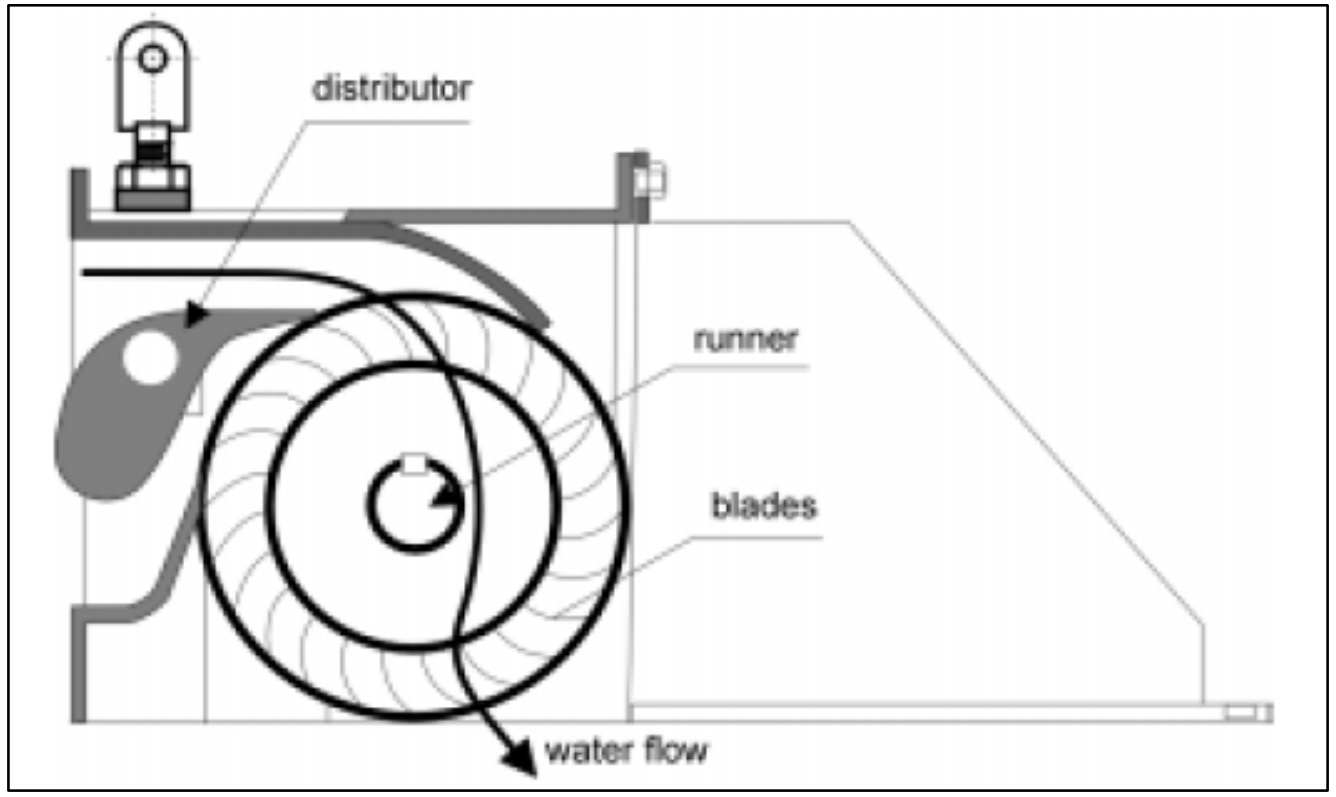

Fig.2. Cross flow turbine. [2] 


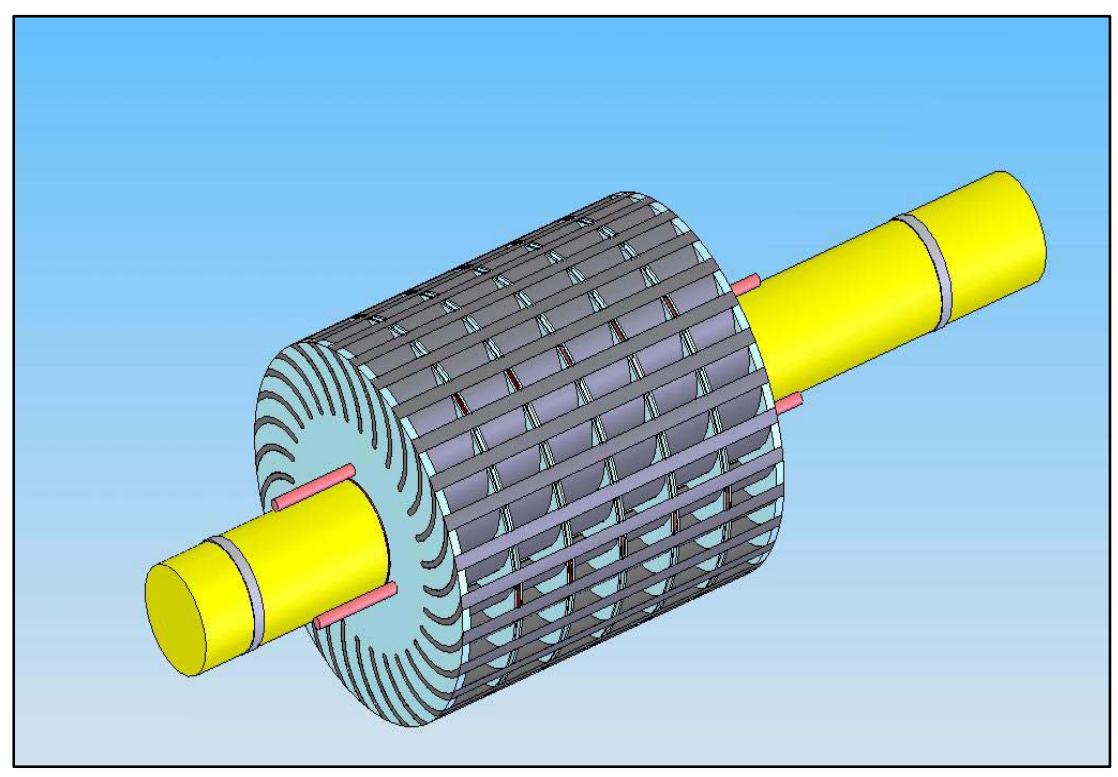

Fig.3. Compact view of cross flow turbine runner

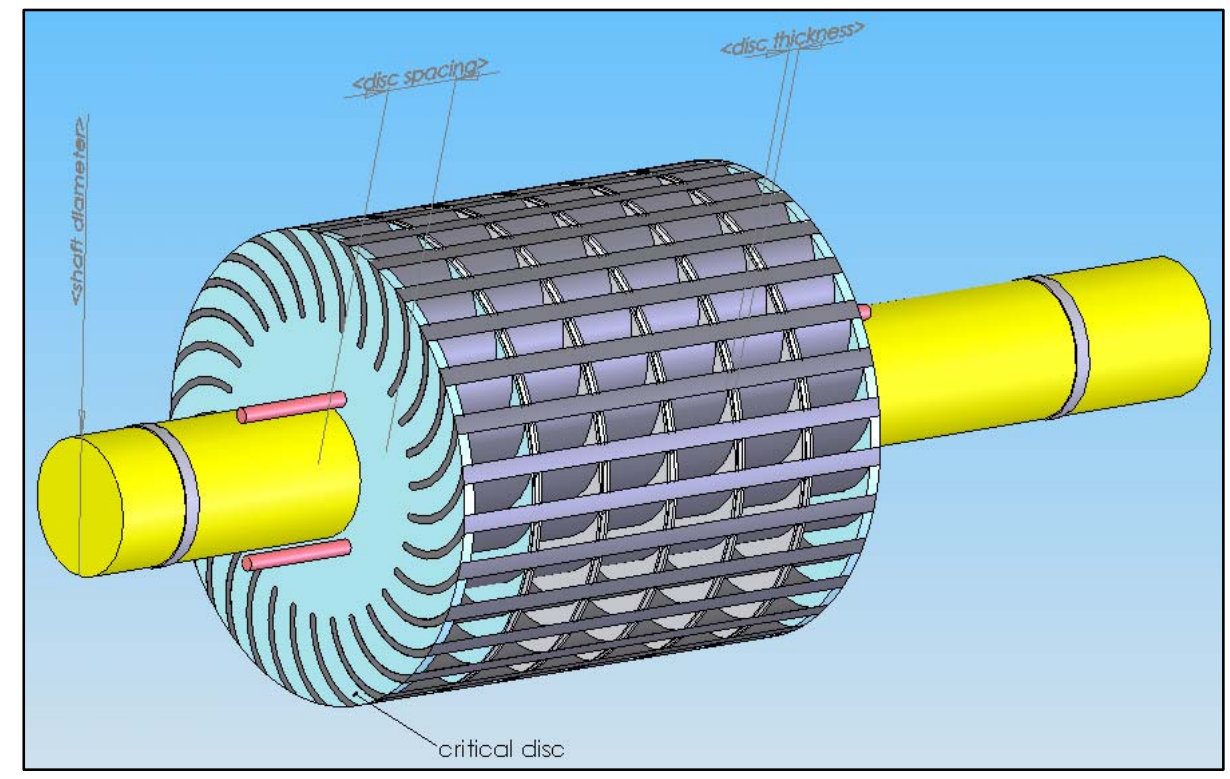

Fig.4. Design variables

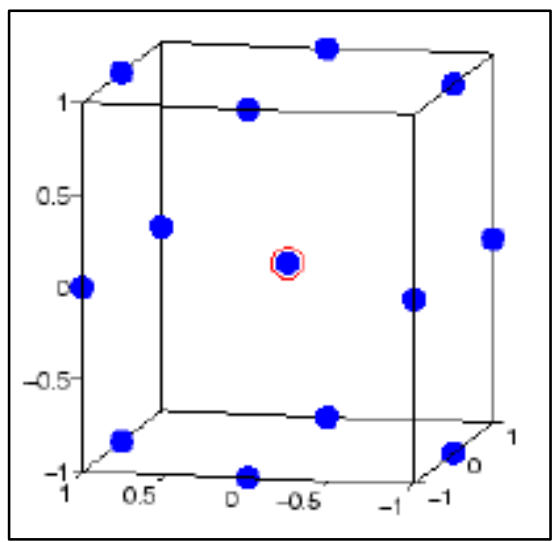

Fig.5. Box-Behnken design algorithm 


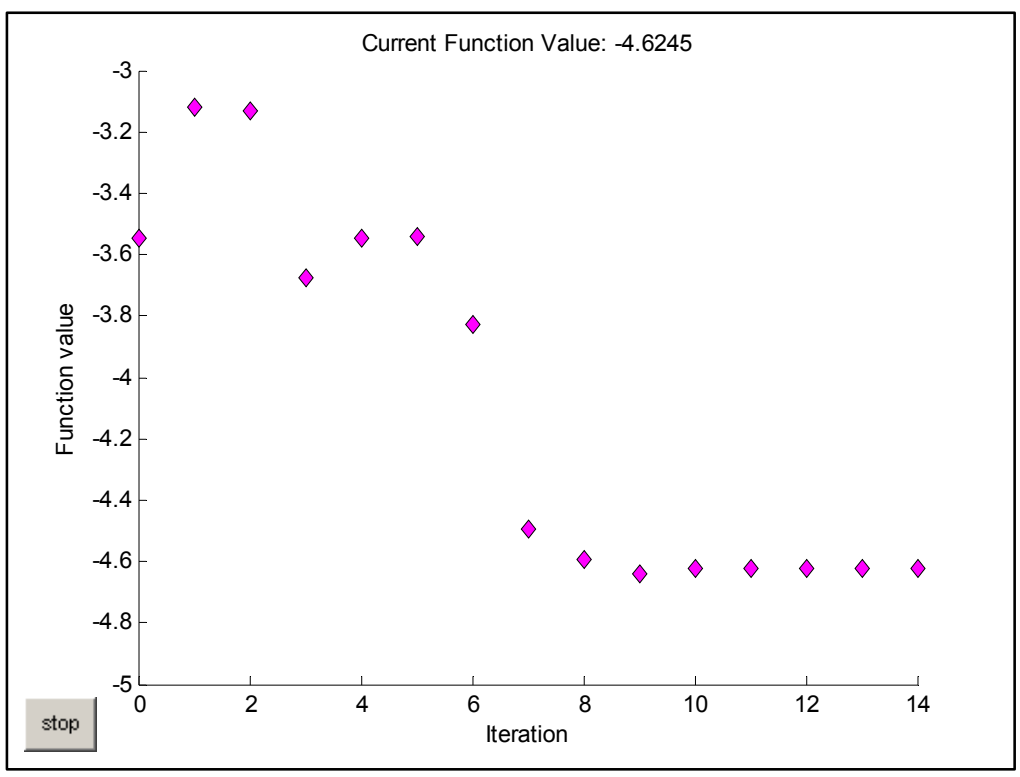

Fig.6. Evolution of SQP algorithm for weight $\leq 50 \mathrm{~kg}$

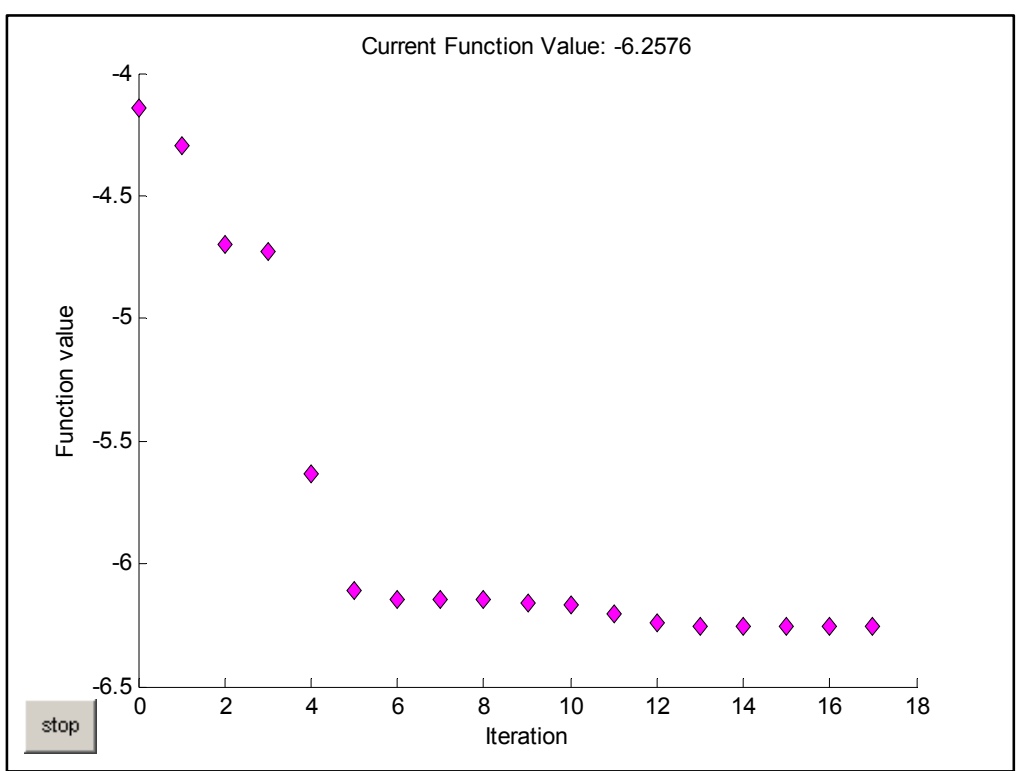

Fig.7. Evolution of SQP algorithm for weight $\leq 60 \mathrm{~kg}$. 


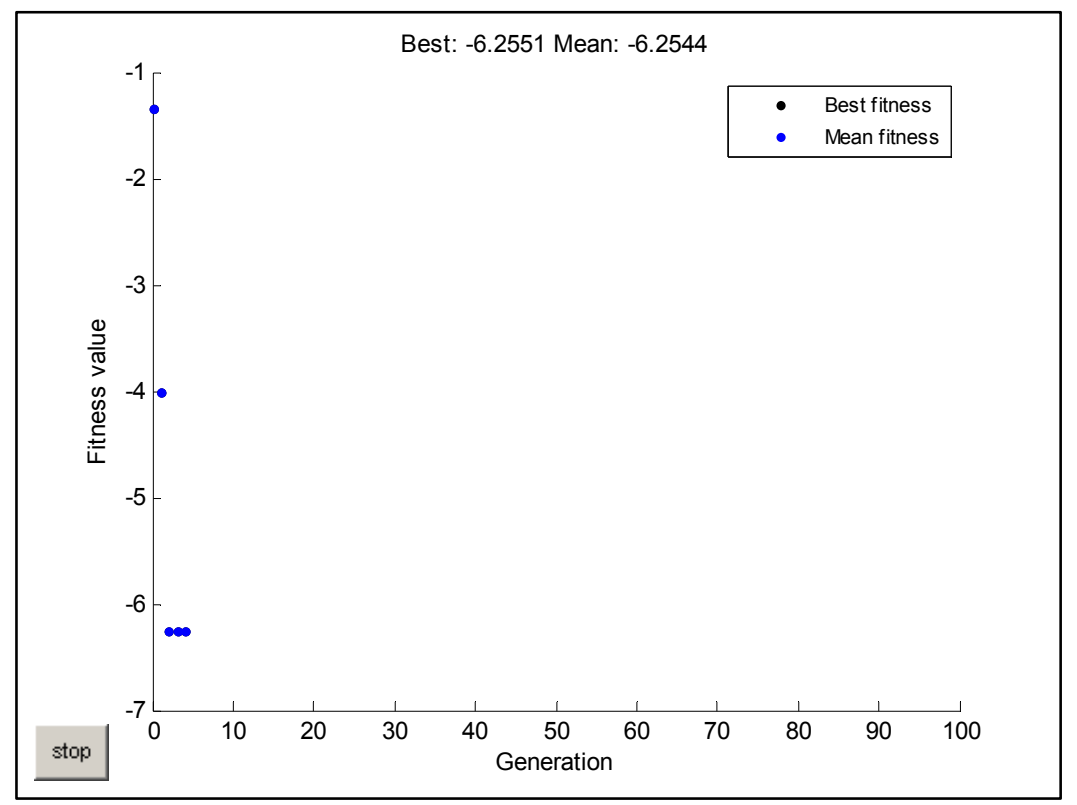

Fig.8. FOS obtained from genetic algorithm for optimization example 1

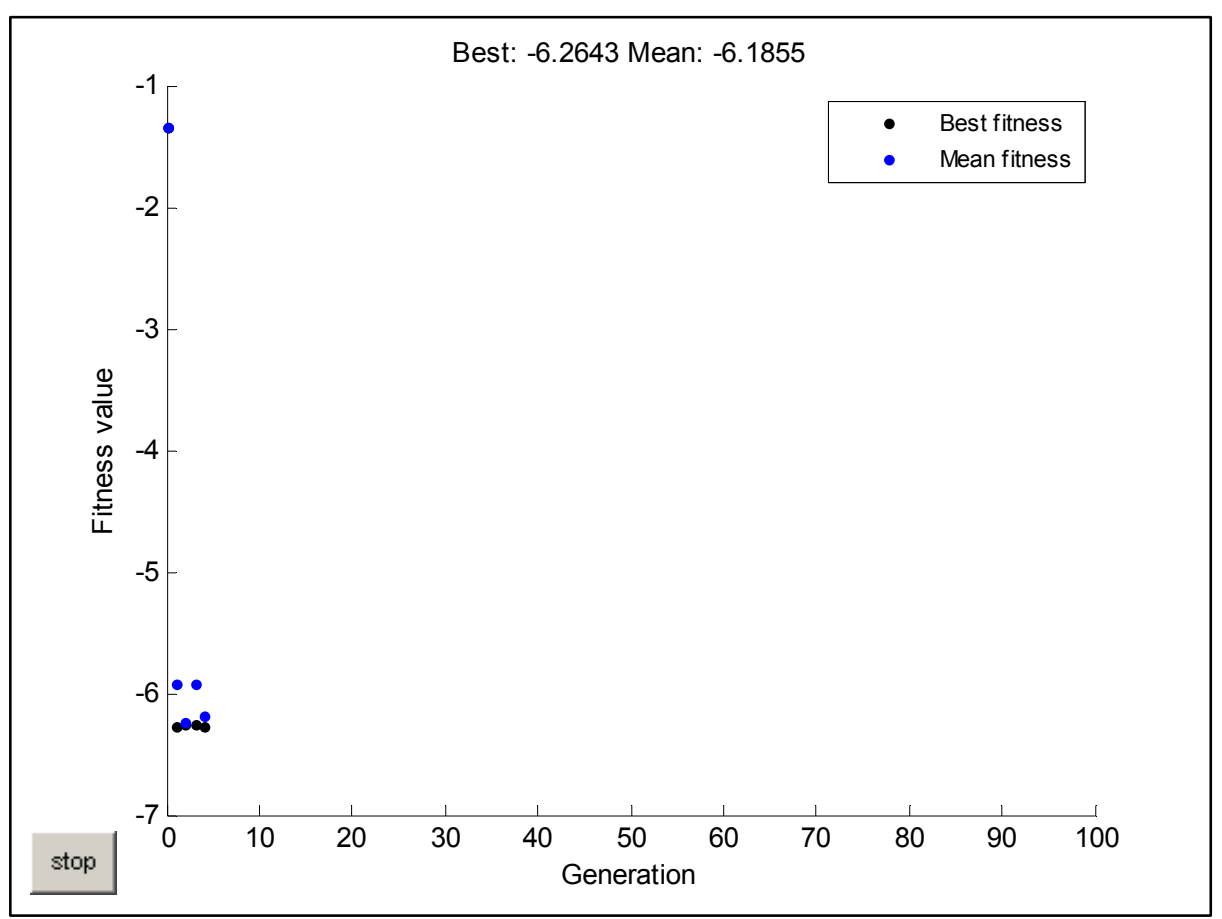

Fig.9. FOS obtained from genetic algorithm for optimization example 2 


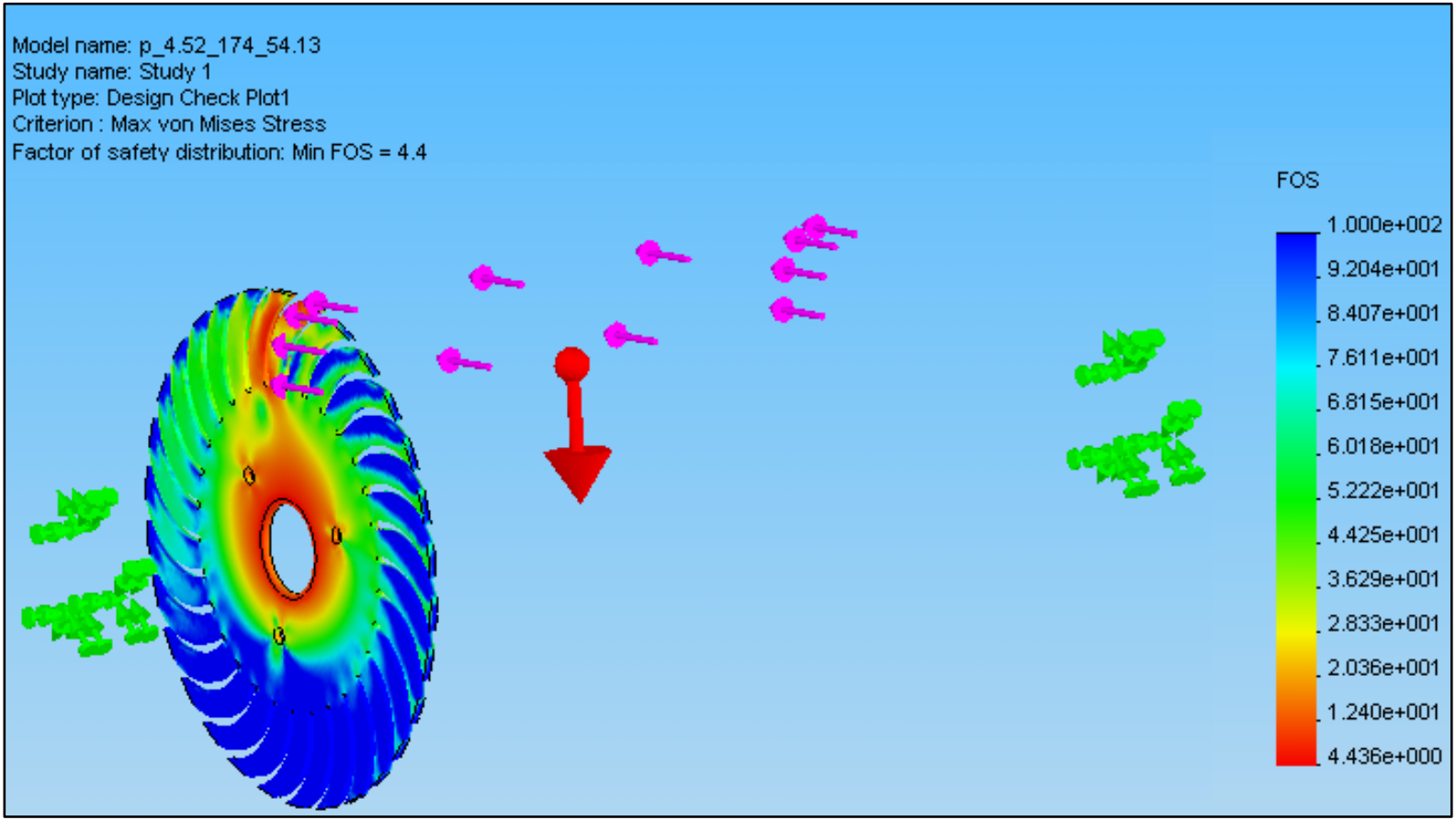

Fig.10. FOS of optimum design by FEA, weight $\leq 50 \mathrm{~kg}$

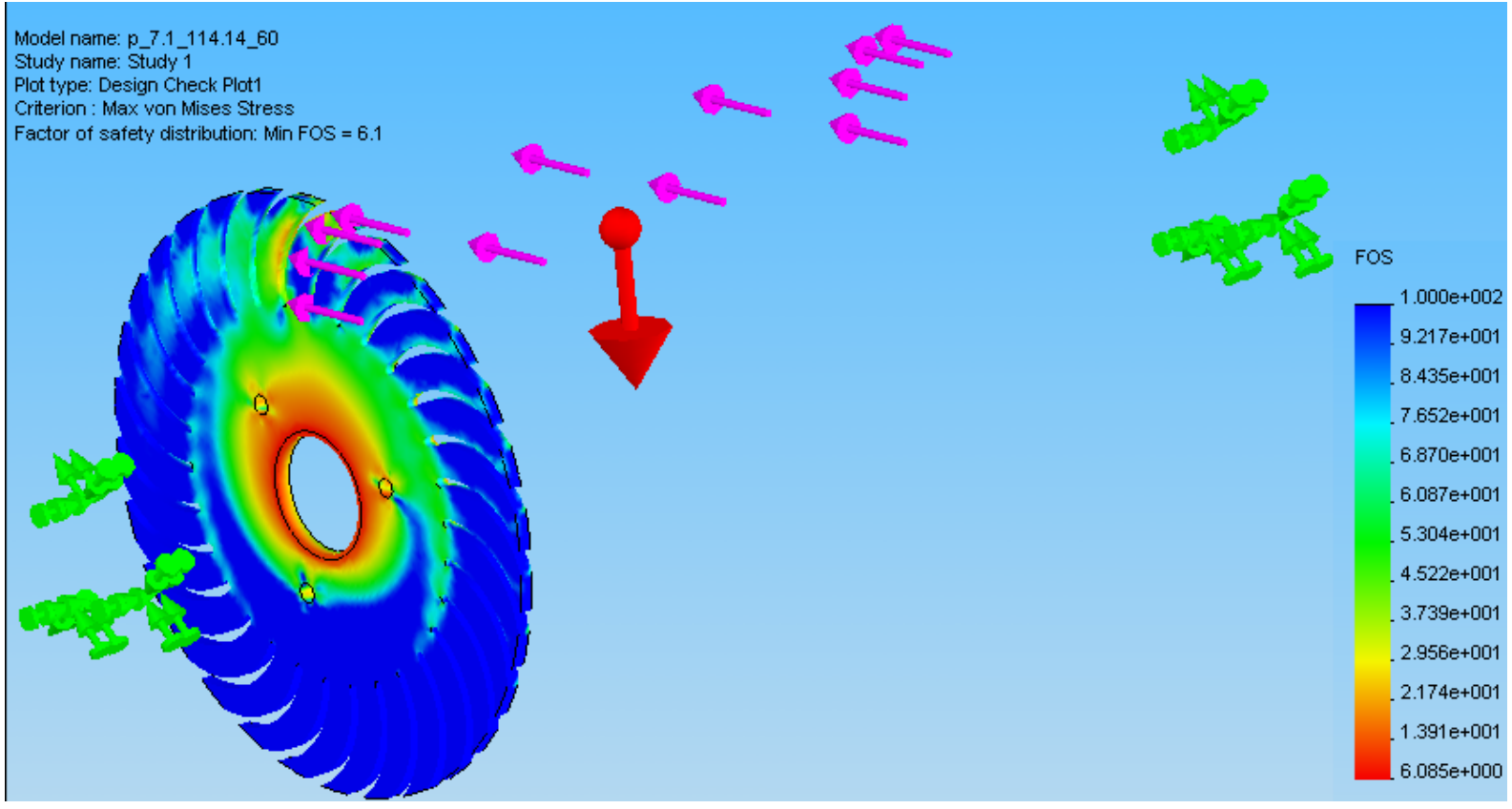

Fig.11. FOS of optimum design by FEA, weight $\leq 60 \mathrm{~kg}$ 